\title{
¿Conviene seguir fomentando las plantaciones forestales en el norte de la Patagonia?
}

\author{
Guillermo E. Defossé
}

Centro de Investigación y Extensión Forestal Andino Patagónico (CIEFAP-CONICET) y Universidad Nacional de la Patagonia San Juan Bosco, Sede Esquel. Chubut, Argentina

\begin{abstract}
RESUMEN. En este ensayo abordo la pregunta sobre la conveniencia (o no) de seguir fomentando las forestaciones en el norte de Patagonia. A partir de elementos de los "servicios ecosistémicos", desarrollado en la Evaluación de los Ecosistemas del Milenio, analizo los aspectos ecológicos, económicos y sociales que responden a esa pregunta. Este análisis contempla las condiciones ecológicas y socioeconómicas actuales de la estepa a forestar y los impactos ecológicos que, a futuro, tendrían las forestaciones sobre algunos atributos estructurales y funcionales de esta estepa. El análisis económico de las forestaciones no sólo arroja beneficios tangibles (creación de riqueza) sino también ambientales (captura y almacenamiento de $\mathrm{CO}_{2}$ ). Socialmente, el efecto sería muy positivo por la generación de empleo que la actividad conlleva. La forestación de áreas degradadas de Patagonia produciría servicios ecosistémicos que beneficiarían a toda la sociedad.
\end{abstract}

[Palabras clave: servicios ecosistémicos, forestaciones, estepa degradada]

\begin{abstract}
AвSTRACT. Is it convenient continuing promoting afforestation in northern Patagonia?: In this study I present an essay to answer the question about the convenience (or not) of continuing promoting afforestation in northern Patagonia. By taking elements of the "Ecosystem Services" concept developed by the Millennium Ecosystem Assessment, I analyzed ecological, economical and social aspects to answer this question. The analysis begins by recognizing the actual ecological and socioeconomical status of the steppe, and the impacts of afforestations on its structural and functional characteristics. In the economic analysis, afforestations will not only provide tangible benefits (goods and services), but also additional environmental benefits (by capturing and storing $\mathrm{CO}_{2}$ ). Socially, the impact would be highly positive, mainly by creating new labor posts generated by this activity. Afforesting degraded areas of Patagonia will produce ecosystem services that will benefit the whole society.
\end{abstract}

[Keywords: ecosystem services, forest plantations, degraded steppe]

\section{INTRODUCCIÓN}

En noviembre de 1976 se realizó en Buenos Aires el Congreso Latinoamericano sobre Celulosa y Papel (INTI 1976), en el que diferentes expertos expusieron argumentos y opiniones acerca del porvenir forestal de Sudamérica y, en especial, el de Argentina. La mayoría de ellos concordó en que ese futuro sería brillante y que Argentina, Brasil y Chile $(\mathrm{ABC})$ liderarían el camino. Estos tres países, cada uno con sus propias características, serían no sólo los grandes proveedores de pulpa de celulosa y papel al mundo en el siglo XXI, sino también de madera y otros productos forestales. Entre ellos, la estrella parecía ser Argentina ya que sus áreas para forestar por fuera de los bosques nativos (en especial, el Delta y la región Mesopotámica) presentaban mejores condiciones ecológicas, de crecimiento, logísticas y de superficies que los de Brasil o Chile. En cuanto a la región Andino-Patagónica, la opinión era casi unánime: no tenía un potencial celulósico- papelero comparable a otras zonas, pero símuy buenas posibilidades para obtener madera y otros productos forestales de calidad.

Treinta y seis años después, en noviembre de 2013, se realizó en Puerto Iguazú, Misiones, el IV Congreso Forestal Argentino y Latinoamericano. Allí se repasó y comparó lo acontecido en los países ABC desde 1976 hasta ese entonces. Brasil y Chile habían logrado un despegue forestal notable. Brasil había exportado productos forestales por 7.97 mil millones de dólares (ABRAF 2012) y Chile por 5.9 mil millones de dólares (INFOR 2014). Mientras tanto, Argentina continuaba luchando por salir del subdesarrollo forestal. Hasta el presente, Argentina es un importador neto que del 2005 al 2011 erogó anualmente entre 227 y 862 millones de dólares para paliar su déficit en productos forestales (SAyDS 2013). Por su parte, Uruguay, que en 1976 no estaba en condiciones de integrar la trilogía $\mathrm{ABC}$, en los últimos 30 años logró conformar un patrimonio forestal importante. 
En la actualidad, ese país exporta productos forestales por 1200 millones de dólares al año y entre 2000 y 2010, su saldo exportable aumentó $1.256 \%$ (SPF 2014).

Ante esta realidad nos preguntamos ¿qué nos pasó durante este tiempo como país en el sector forestal? ¿Qué impidió que nos transformáramos en esa potencia forestal vaticinada en 1976? ¿Cómo puede ser que teniendo superficies y ventajas ecológicas, económicas y productivas no hayamos paliado aún el déficit crónico en productos forestales? Quizás sea importante señalar que algunas provincias y muchos sectores hicieron y continúan haciendo grandes esfuerzos para lograr nuestro despegue forestal. Sin embargo, en el nivel de país, dichos esfuerzos han resultado insuficientes: el déficit se agrava año tras año.

¿Qué pasó desde ese entonces en la Patagonia Andina? ¿Cómo evolucionó el sector forestal? ¿Qué hicimos para continuar con la tarea pionera del Instituto Forestal Nacional (IFONA) en estaciones experimentales de Patagonia a fin de comenzar a producir productos forestales de calidad? ¿Qué cosas ocurrieron para que en 2015 nos preguntemos cuán conveniente es seguir fomentando las plantaciones forestales en Patagonia? Con un territorio de casi $750000 \mathrm{~km}^{2}$ y sólo 900 km² forestados $(0.12 \%$ de su superficie) (Loguercio et al. 2008; Defossé et al. 2014): ¿cuál es la razón profunda, o las motivaciones reales, para que todavía nos hagamos la misma pregunta? La respuesta sólo puede emerger de un análisis profundo, desapasionado y abarcativo de todos los pros y los contras de propiciar o desaconsejar el fomento de esta actividad incipiente. Sería ingenuo responder a la pregunta desde una concepción meramente ecológica ya que encierra aspectos económicos y sociales, y posee un contenido político que trasciende lo ecológico, lo económico y lo social. Entonces, las respuestas que deriven de esta pregunta — sobre todo viniendo del sector académico y de investigación-pueden tener efectos variados, en particular entre quienes deben tomar decisiones políticas que implican continuar, o no, con la promoción de la actividad forestal en la Patagonia.

Por esas razones, este análisis se debe basar no sólo en información ecológica previa sino también en los factores económicos y sociales asociados a esta actividad. Es clave desarrollarlo a partir de elementos contenidos en el documento Evaluación de los Ecosistemas del Milenio (MEA 2005). Esta evaluación ha sido diseñada para "satisfacer las necesidades que tienen los responsables de la toma de decisiones y el público general, de información científica acerca de las consecuencias de los cambios en los ecosistemas para el bienestar humano y las opciones para responder a esos cambios" (MEA 2005). Este foro, "Ecología y Sociedad", constituye una oportunidad excelente para presentar distintas visiones que aporten a la discusión sobre el tema. También le servirá de insumo a los responsables de formular políticas y de tomar decisiones sobre el tema, decisiones cuyas consecuencias trascenderán nuestra generación y las que nos sucedan.

\section{La actividad forestal en la Patagonia Andina}

Desde la colonización europea y hasta poco tiempo atrás, la ganadería ovina (Defossé \& Robberecht 1987) y la actividad forestal basada sobre el aprovechamiento del bosque nativo (Rothkugel 1916; Jovanovski et al. 2003) fueron los pilares del desarrollo económico de la Patagonia Andina Central (i.e., provincias de Neuquén, Río Negro, Chubut y, en menor medida, Santa Cruz). En el centro-norte de Neuquén, la araucaria araucana (Araucaria araucana [Molina] K. Koch), el raulí (Nothofagus alpina [Poepp. \& Endl.] Oerst.) y el roble pellín (Lophozonia obliqua [Mirb.] Oerst.) fueron las principales especies aprovechadas hasta la década del sesenta del siglo pasado. Entre San Martín de los Andes - Neuquén-y Corcovado - Chubut-, el ciprés de la cordillera (Austrocedrus chilensis [D. Don] Pic. Serm. \& Bizzarri) y la lenga (Nothofagus pumilio [Poepp. \& Endl.] Krasser) fueron relevantes para la construcción (e.g., tirantería, revestimientos, aberturas, etc.) y la fabricación de muebles. Sin embargo, en los últimos años, las áreas de bosque nativo aprovechadas en esta región disminuyeron de manera notable (SSBCh 2014). La disminución se debió a la herbivoría en el bosque (Urretavizcaya et al. 2012; Quinteros 2014), a los incendios (Veblen et al. 2008; Defossé et al. 2014), a enfermedades como el mal del ciprés (Greslebin \& Hansen 2010), al avance de la construcción en las áreas de interfaz urbano-rural (Defossé et al. 2014) y a las medidas de protección. Hasta mediados de los años noventa del siglo pasado, la Patagonia Andina Central se autoabastecía y hasta exportaba madera de especies nativas. Hoy se ha convertido en una importadora 
neta de productos forestales. Estadísticas del Chubut (SSBCh 2014) indican que en 1994 se aserraron $25000 \mathrm{~m}^{3}$ de lenga y sólo 1800 $\mathrm{m}^{3}$ en 2012. En cuanto al ciprés, en 1997 se procesaron $15000 \mathrm{~m}^{3}$, que se redujeron a 5000 $\mathrm{m}^{3}$ en 2012. En cuanto a la madera proveniente de forestaciones, en 1997 se aserraron 2000 $\mathrm{m}^{3}$, que crecieron a $25000 \mathrm{~m}^{3}$ en 2012; este incremento sólo permitió satisfacer el 12\% del consumo de madera de machimbre y el $28.8 \%$ de tablas y tirantes en Chubut (Salvador et al. 2007). Hoy, más del $85 \%$ de la demanda de machimbre, del 70\% de tirantes y tablas y el $100 \%$ de tableros y paneles comercializados en Neuquén, Río Negro y Chubut es satisfecha por productos extra-regionales (en particular, de Misiones y Chile). Esto implica un balance socioeconómico y ecológico negativo que eroga divisas (al dejar de producirse bienes locales) y genera una huella de carbono negativa al transportarse madera desde casi 3000 km (Misiones) ó 1000 km (Chile).

\section{Las forestaciones con especies exóticas en la Patagonia}

A mediados de 1970 se implementaron incentivos impositivos para fomentar, en toda Argentina, las forestaciones con especies de rápido crecimiento. El objetivo fue paliar el déficit de celulosa y de papel (PEN 1977). En la Patagonia Andina, esos incentivos y los buenos rendimientos obtenidos en estaciones experimentales del IFONA desde 1940 estimularon forestaciones que reemplazaron el bosque nativo, y en especial el bosque mixto de ciprés y coihue (Nothofagus dombeyi [Mirb.] Oersted) (Dezotti \& Sancholuz 1991; Simberloff et al. 2002; Amoroso \& Larson 2010). Las plantaciones mostraron buenas tasas de crecimiento, pero se realizaron sin criterios silviculturales ya que su objetivo era sólo la producción de celulosa. Desde diferentes sectores de la sociedad, y de acuerdo con la tendencia internacional hacia una silvicultura más natural (Coates \& Burton 1997; O'Hara 2001), en las décadas del ochenta y noventa se comenzó a desalentar aquellas plantaciones que reemplazaran bosques nativos. Las evidencias científicas indicaban que las forestaciones monoespecíficas, plantadas a densidades altas, creaban ambientes artificiales que modificaban los atributos estructurales y funcionales (Sarasola et al. 2006) y los servicios ambientales que prestaban los bosques reemplazados. Desde entonces, en Patagonia, ninguna plantación forestal reemplaza bosques nativos.

En los años noventa, estudios ecológicos, edafológicos y productivos relevaron y delimitaron el potencial de la estepa patagónica adyacente al bosque nativo para realizar forestaciones con coníferas exóticas y otras especies de calidad (Colmet Daage et al. 1995; Urzúa 1991; Godoy et al. 2007). Este potencial, de casi 2000000 ha (Irisarri \& Mendía 1991; Irisarri et al. 1995; Loguercio et al. 2004) (Figura 1), está basado en las condiciones agroecológicas y climáticas de esta estepa. Allí juegan un papel importante las cenizas volcánicas, que forman un horizonte en el

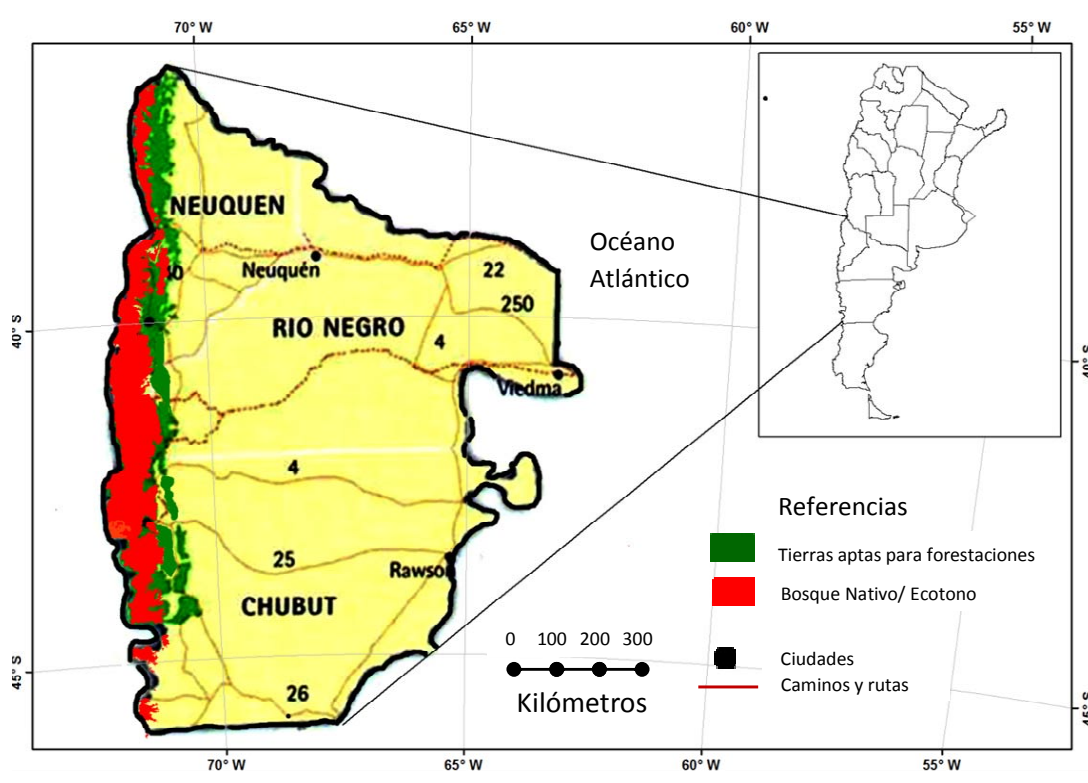

Figura 1. Área forestable de la estepa en Patagonia Central (en verde). De ésta, el $40 \%$ tiene condiciones muy favorables para forestarse con coníferas de rápido crecimiento (basado en Colmet Daage et al. 1995 y Loguercio \& Decechis 2006a,b).

Figure 1. Afforestable area in the Patagonian steppe (in green). Of this, about $40 \%$ showed very high favorable conditions to be afforested with fast-growing conifers (based upon Colmet Daage et al. 1995 and Loguercio \& Decechis 2006a,b). 
subsuelo. Estas cenizas contienen sustancias alofánicas (Buduba 2006) capaces de retener humedad y de proveerla a las plantaciones durante el déficit primavero-estival (Colmet Daage et al. 1995; Buduba 2006). Las raíces de las plantas de la estepa no acceden a estas cenizas por ocupar horizontes más superficiales. Sin embargo, los condicionantes ecológicos (e.g, relictos, parches de estepa que se quieran conservar, corredores de biodiversidad, áreas de reserva, mallines, áreas ribereñas y rocosas, etc.) y económicos (e.g., cercanías a caminos, áreas urbanas y periurbanas, etc.) reducirían esta superficie a 800000 ha (Loguercio \& Dececchis 2006 a, b). Además, las forestaciones en esta estepa tendrían ventajas comparativas y competitivas para desarrollarse dentro del Mecanismo de Desarrollo Limpio (CDM) del Protocolo de Kioto (Loguercio et al. 2004). Aparte del bajo costo de la hectárea, la vegetación de esta estepa está deteriorada por sobrepastoreo: comparada con otros ecosistemas, almacena muy poca biomasa tanto en la parte aérea como en la radical (la línea base) (Colmet Daage et al. 1995; Defossé et al. 2011). Esto permite que las forestaciones tengan una alta adicionalidad no sólo como almacenadoras de carbono (dentro del CDM) sino también por sus tasas de crecimiento elevadas, iguales o superiores a las que presentan en sus sitios de origen (Urzúa 1991; Gonda \& Lomagno 1995; Gonda 2005). La adicionalidad elevada y el excelente crecimiento hace que estas forestaciones resulten muy atractivas como un servicio ecosistémico (MEA 2005), ya que podrían, además, proveer productos forestales, generar riqueza y emplear mano de obra en una región con alta desocupación.

\section{Impactos ecológicos, económicos, y sociales de las forestaciones en Patagonia}

¿Cuáles serían los impactos ecológicos, económicos y sociales más importantes, tanto positivos como negativos, que producirían las forestaciones en Patagonia? Mucho se ha escrito sobre los impactos ecológicos sobre los ecosistemas donde se las implanta. Se ha escrito mucho menos sobre estos impactos desde lo económico y/o lo social. Por eso, el análisis de este ensayo está inspirado en el concepto de "servicio ecosistémico", mencionado previamente y desarrollado en profundidad en la Evaluación de los Ecosistemas del Milenio (MEA 2005). Para hacer este análisis es necesario conocer de antemano el estado estructural y funcional actual de la estepa donde se propone forestar. Es importante recordar que esta estepa no es prístina ni mucho menos, ni tampoco las forestaciones la estarían reemplazando por completo. La estepa tiene una historia de 100 años de uso intensivo y de deterioro por sobrepastoreo ovino, como lo atestigua la literatura científica desde los años cincuenta (Soriano 1956; Ares et al. 1990; Bertiller \& Defossé 1990; Defossé et al. 1990). Durante los años ochenta y noventa del siglo pasado se realizaron ingentes esfuerzos en investigación para comprender la estructura y el funcionamiento de estos ecosistemas y para proponer alternativas que permitieran su recuperación (proyectos PRECODEPA y LUDEPA, INTA 2010). Aunque la información generada fue prolífica, en la mayoría de los casos no fue posible revertir el deterioro. En los últimos 20 años, varios factores socioeconómicos y ecológicos adversos (e.g., la baja del precio internacional de la lana, la caída en la demanda de mano de obra rural y las sequías prolongadas) afectaron la actividad ovina y el deterioro del recurso pasturil (González 2000; De Jong 2007). En el último decenio, la ganadería ovina para los pequeños productores pasó de medianamente lucrativa a de subsistencia. Como consecuencia de la falta de trabajo rural, muchos trabajadores debieron migrar a la periferia de ciudades costeras y andinas a fin de conseguir una "changa" o un subsidio estatal para poder sobrevivir. Aquellos ganaderos que pudieron paliar la situación (por tener predios más grandes y con mejores condiciones agroecológicas) fueron cambiando la ganadería ovina por la bovina, y recientemente sumaron la producción de carne en "feed-lots". Estos feed-lots requieren menos personal que la ganadería ovina y son mucho más lucrativos. El paulatino despoblamiento humano de los campos, acelerado desde la sequía del 2005 al 2009, generó impactos socioeconómicos nunca antes vistos en Patagonia (aliviados en parte por subsidios estatales). Como ejemplo del deterioro, Chubut pasó de 5 millones de ovejas en 2005 a 2.8 millones en 2012, lo que representó una merma del 55\% en su stock ovino (DGEyCCh 2012). Los departamentos Cushamen, Futaleufú, Languiñeo, Tehuelches y Río Senguer (los más productivos para la ganadería ovina del Chubut y con áreas aptas para forestar) perdieron $60 \%$ de sus ovinos —de 2.26 millones a 1.36 millonesentre 2005 y 2012. Esta caída también se reflejó en los departamentos colindantes 
Pilcaniyeu, Bariloche y Norquinco (provincia de Rio Negro). El deterioro de los campos es difícil de revertir con el esquema actual de monoproducción ovina. Desde el punto de vista socioeconómico, se necesitan alternativas que cambien esta matriz productiva (i.e., mediante forestaciones) y que generen riqueza y bienestar. Sin embargo, queda pendiente la pregunta: desde lo ecológico, ¿cómo impactarían las forestaciones sobre algunos atributos de esta estepa como la biodiversidad, la combustibilidad del paisaje o la capacidad invasora de alguna de las especies introducidas?

\section{Impactos sobre la diversidad, combustibilidad y capacidad invasora de las forestaciones}

En esta estepa es difícil encontrar lugares de referencia en donde la diversidad no haya sido afectada. Por ese motivo debemos ser muy cautos al generalizar los impactos de las forestaciones sobre la diversidad. Hoy se acepta que la densidad inicial de las plantaciones juega un rol fundamental en la magnitud de estos impactos (Lantschner \& Rusch 2014). La disminución de la diversidad y la fragmentación del ambiente es evidente en aquellas forestaciones densas que reemplazaron al bosque nativo (Pauchard et al. 2008; Orellana 2011). En cambio, plantaciones jóvenes ( 20 años) realizadas en la estepa, raleadas a baja densidad, presentaron impactos leves sobre la diversidad vegetal (Godoy \& Defossé 2007). Lantschner \& Rusch (2014) también determinaron que los impactos sobre la diversidad de la fauna en general se atenúan cuando las forestaciones son ralas. Otro estudio demostró que el ensamble de aves es similar tanto en el bosque nativo como en forestaciones adultas de Neuquén (Pescador \& Peris 2014). Comparadas con forestaciones densas, las bajas densidades de plantación (i.e., en etapas iniciales o por podas y raleos) permiten una mayor entrada de luz y una mejor distribución de la precipitación hacia el estrato herbáceo; por lo tanto, impactan menos sobre la biodiversidad. Varios trabajos han profundizado sobre las forestaciones como modificadoras del ciclo del agua (Buduba 2006; Fernández et al. 2007; Licata et al. 2011). La mayoría de estos trabajos corresponden a áreas forestadas en bosques nativos $\mathrm{y}$, aunque tienen gran valor indicativo, no se los puede extrapolar a áreas de estepa ya que en su mayoría son pseudorepeticiones (Licata et al. 2011). En la estepa, las plantas nativas y las plantaciones pueden desarrollarse y convivir sin inconvenientes al usar agua de distintos horizontes (Fernández et al. 2008). Por ejemplo, en comparación con la estepa, la gramínea palatable Festuca pallescens (St. Yves) Parodi, a iguales valores de precipitación y luz mejora sus relaciones hídricas y aumenta su crecimiento bajo el dosel de pinos (Fernández 2003). Esto abre la posibilidad de desarrollar sistemas silvopastoriles, otro "servicio ecosistémico" que diversificaría la producción y agregaría riqueza a la estepa degradada y con escasas alternativas de desarrollo.

En cuanto a la capacidad invasora de las coníferas introducidas en Patagonia, el 80\% de las forestaciones corresponden a pino ponderosa, considerado por sus características como "no invasor" (Simberloff et al. 2002). Del resto, los pinos oregón, radiata y contorta podrían considerarse como invasores (Pauchard et al. 2008; Orellana 2011), aunque en áreas de estepa se los puede controlar con un manejo adecuado de las forestaciones. En cuanto a la combustibilidad del paisaje, todas las especies a forestar han co-evolucionado con el fuego como disturbio natural (Wright \& Bailey 1982; Agee 1993), como también ha sucedido con las especies de la estepa patagónica (González 2011; Defossé et al. 2014). La clave para desarrollar forestaciones con bajo riesgo de incendios está en manejar bien la plantación, incluyendo podas y raleos y el tratamiento de los restos con tecnologías adecuadas (e.g., triturado, quemas prescriptas, etc.) (Defossé et al 2011; Godoy et al. 2013; Defossé et al. 2014). Como corolario, es necesario profundizar más las investigaciones sobre los impactos de las forestaciones en la estepa, sin perder de vista que aun forestando las 800000 ha mencionadas anteriormente se cubriría sólo el 1.54\% del territorio de las provincias de Neuquén, Río Negro y Chubut. En ese contexto y a esa escala, es probable que las forestaciones en Patagonia tengan un impacto ecológico escaso o nulo, mientras que los impactos económicos y sociales resultarían positivos y alentadores (Gyenge et al. 2010).

\section{Destino de las plantaciones}

La pregunta inicial, ¿conviene (o no) seguir fomentando las plantaciones forestales en el norte de la Patagonia?, podría conducirnos a otra: en caso de seguir fomentándose, ¿qué haríamos con sus productos y subproductos? 
Al descartar el destino celulósico-papelero, la respuesta sería: paliar el $85 \%$ del déficit en machimbres, el $70 \%$ en tirantería y tablas o el $100 \%$ en tableros y paneles usados en Patagonia, que hoy provienen de mercados extra-regionales. Esta trasformación primaria de la madera daría muy pronto un gran impulso a la foresto-industria, y crearía puestos de trabajo genuinos y de calidad. Esto podría aliviar la desocupación actual o la dependencia de planes sociales y mejoraría la economía de la región al recircular internamente el dinero proveniente del trabajo y el ahorro de divisas. A partir de datos muy conservadores, se puede estimar que las forestaciones y su industria asociada generan entre 1 y 5 puestos de trabajo estable por cada 10 ha forestadas (AFOA 2014: SPF 2014; Alonso Schwarz et al. 2015). Si se forestaran las 800000 ha identificadas como aptas, se generarían 80000 empleos (directos e indirectos). Esto equivaldría a $24.6 \%$ de la población total o a más de $50 \%$ de la población económicamente activa que hoy habita los departamentos andinos de Neuquén, Río Negro y Chubut (donde se proponen las forestaciones), ocupando sólo el $5.3 \%$ de su superficie (INDEC 2010).

Otro aspecto quizá menos conocido por el público en general sobre el destino de las forestaciones son los desarrollos tecnológicos realizados por el Área de Tecnología de la Madera del CIEFAP. Este área, de cuyos logros deberíamos estar orgullosos todos los patagónicos, ha generado una batería formidable de manufacturas a partir de maderas, raleos y otros subproductos de forestaciones de Patagonia. Éstas comprenden desdejuguetes, sillas, revestimientos y muebles hasta casas-prototipo realizadas con paneles a base de raleos de pino. Dichas casas llevan más de un decenio de construidas y han resultado muy confortables y térmicas ya que los paneles en sus paredes superan el coeficiente térmico de los ladrillos de uso común en la región. Al poseer una tecnología sencilla se las puede confeccionar en carpinterías con mediana infraestructura. Su producción masiva permitiría reducir una parte del déficit habitacional de la Patagonia Andina, además de ahorrar energía y disminuir la emisión de gases con efecto invernadero. Esto es así ya que la madera que se usa para construirlas retiene $\mathrm{CO}_{2}$ en su estructura (mientras que la fabricación de los materiales tradicionales, ladrillos, emite $\mathrm{CO}_{2}$ [el proceso inverso]). Como vemos, forestar la estepa patagónica no sólo tendría ventajas económico-sociales, sino también ecológicas al contribuir a la reducción de las emisiones globales de $\mathrm{CO}_{2}$.

\section{Consideraciones FinAles}

En la Evaluación del Milenio (EM 2005) quedó explícito que el concepto de "servicio ecosistémico" se refiere a los beneficios que los ecosistemas brindan a la sociedad. Aplicarlo a casos reales requiere tanto tener en cuenta sus costos y beneficios como identificar y diferenciar los grupos sociales que se beneficiarán o perjudicarán económicamente con su desarrollo (Daw et al. 2011). En el caso de fomentar las forestaciones en Patagonia, los grupos que se beneficiarían en el corto plazo serían, naturalmente, los plantadores forestales (muchos desocupados rurales que requieren hoy de asistencia social para subsistir), los viveristas y los pequeños empresarios plantadores, los productores cuyos campos perdieron receptividad y los comerciantes de localidades pequeñas. En el mediano plazo se beneficiarían quienes se dediquen a la foresto-industria, al comercio maderero o agropecuario y a toda la cadena productiva que dinamizan las forestaciones. En el corto plazo, las forestaciones no impactarían sobre el grupo de aquellos que obtienen sus ingresos del erario público (nacional, provincial o municipal), aunque sí en el largo plazo al generarse riqueza y bienestar para toda la sociedad. En este esquema es difícil encontrar efectos negativos sobre la sociedad o el ecosistema que se forestará. Como lo hicieron anteriormente Brasil, Chile y Uruguay, las forestaciones en Patagonia pueden crear riqueza, generar empleos genuinos y diversificar la producción de una estepa degradada sin otras alternativas productivas. Todo esto mejoraría la calidad de vida de sus habitantes. Para que ocurra, no sólo debemos contar con políticas de Estado claras, estables y sostenibles, sino también logar que se cumplan a rajatabla. De nada sirve si los subsidios destinados a forestar no llegan en tiempo y forma a sus beneficiarios; sus demoras en el pasado reciente han causado el quiebre en la cadena de pagos y la desaparición de muchos actores ligados a la actividad. Asimismo, es imprescindible que las instituciones administrativas y de investigación apoyen con fuerza la actividad mediante trabajos que mejoren la productividad y que ayuden a conservar la diversidad de la estepa. Por último, y dando 
por cumplidas las premisas anteriores, es necesario que los empresarios apuesten al sector, capitalizando las enormes ventajas que ofrecen las forestaciones en Patagonia. Si así lo hacemos, las generaciones venideras nos lo agradecerán.

\section{BiBLIOGRAFÍA}

Agee, JK. 1993. Fire Ecology of Pacific Northwest forests. Island Press, Washington D.C.

Alonso Schwarz, G; C Burg \& J Cuevas. 2015. Impacto de los Bosques de Cultivo. Importancia socioeconómica y efecto multiplicador. Fundación Mediterránea. (DOI) http:// www.ieral.org/images_db/noticias_archivos/3104Impacto\%20Cultivo.pdf.

Amoroso, MM \& BC Larson. 2010. Stand development patterns as a consequence of the mortality in Austrocedrus chilensis forests. Forest Ecol. Manage., 259:1981-1992.

Ares, JO; AM Beeskow; MB Bertiller; CM Rostagno; MP IRISARRI; ET AL. 1990. Structural and dynamic characteristics of overgrazed grasslands of northern Patagonia, Argentina. Pp. 149-175 en: Breymeyer, A (ed.). Managed Grasslands, Regional Studies. Vol 17 A. Elsevier, Amsterdam.

Ascociación Forestal Argentina. 2014. Bosques de cultivo. Boletín Afoa. http://www.afoa.org.ar/bosques_en_ argentina_detalle.php? $\mathrm{p}=41$.

Associaçao Brasileira de Produtores de Florestas Plantadas (ABRAF). 2012. Anuário estatístico da ABRAF 2012 ano base 2011. ABRAF, Brasília. Pp. 149.

Bertiller, MB \& GE Defossé. 1990. Grazing and plant growth interactions in a semiarid Festuca pallescens grassland. J. Range Manage., 43:300-303.

Buduba, CG. 2006. Modificaciones en el pH y contenido de materia orgánica en suelos del ecotono estepa / bosque andino patagónico por implantación de pino ponderosa. Tesis Doctoral. Facultad de Agronomía, Universidad de Buenos Aires.

CoATes, KD \& PJ Burton. 1997. A gap-based approach for development of silvicultural systems to address ecosystem management objectives. For. Ecol. Manage., 99:337-354.

Colmet Daage, F; M Lanciotti \& A Marcolin. 1995. Importancia forestal de los suelos volcánicos de la Patagonia Norte y Central, Climo-topo-secuencias de suelos y vegetación. ORSTOM (Francia)-INTA Bariloche, Argentina. Consejo Federal de Inversiones, Argentina.

DAw, T; K BRown; S Rosendo \& R POMEROY. 2011. Applying the ecosystem services concept to poverty alleviation: the need to disaggregate human well-being. Env. Conserv., 38:370-379.

Defossé, GE \& R Robberecht. 1987. Patagonia: range management at the end of the world. Rangelands, 9:106-109.

Defossé, GE; MB Bertiller \& JO Ares. 1990. Aboveground Phytomass Dynamics in Grassland Steppe of Patagonia, Argentina. J. Range Manage., 43:157-160.

Defossé, GE; GA Loguercio; FJ Oddi; JC Molina \& PD Kraus. 2011. Potential CO2 emissions mitigation through forest prescribed burning: a case study in Patagonia, Argentina. For. Ecol. Manage., 261:2243-2254.

Defossé, GE; MM Godoy; LO Bianchi; NS Lederer \& C
KUNST. 2014. Fire history, fire ecology and management in Argentine Patagonia: from ancient times to nowadays. In: Alexander, M and B Leblon (eds.). Current International Perspectives on Wildland Fires, Mankind and the Environment. Nova Science Publishers. New York (en prensa).

De Jong, G. 2007. Desertificación en la Patagonia: análisis para la acción. Departamento de Geografía, Facultad de Humanidades de la Universidad Nacional de Comahue. Neuquén.

Dezzotti, A \& L Sancholuz. 1991. Los bosques de Austrocedrus chilensis en Argentina: ubicación, estructura y crecimiento. Bosque, 12(2):43-52.

Dirección General de Estadísticas y Censos del Chubut (DGEYCCH). 2012. Estadísticas ganaderas 2005-2012. Dir. Gral. de Ganadería. Rawson, Provincia del Chubut.

FERNÁNDEZ, ME. 2003. In uencia del componente arbóreo sobre aspectos fisiológicos determinantes dela productividad herbácea en sistemas silvopastoriles de la Patagonia Argentina. Tesis Doctoral, Universidad del Comahue, Bariloche, Argentina.

FernándeZ, ME; JE Gyenge; J Licata; T Schlichter \& B Bond. 2007. El uso de isótopos del H y O para determinar fuentes de agua e interacciones ecológicas entre especies del NO de la Patagonia. En: Lázzari, MA \& C Videla (eds.). Empleo de Isótopos estables en agroecosistemas. Universidad Nacional del Sur, Bahía Blanca, Argentina.

Fernández, ME; JE Gyenge; J Licata; T Schlichter \& B BOND. 2008. Belowground interaction between tres and grasses in a temperate semiarid agroforestry system. Agroforestry Systems, 74:185-197.

Godoy, MM \& GE Defossé. 2007. Impacto de las forestaciones de pino sobre algunos atributos de la comunidad vegetal nativa en Patagonia. Pag. 103 en: Actas de la Primera Reunión sobre Forestación en la Patagonia. Esquel, Chubut, 25 al 27 de abril de 2007.

Godoy, MM; GE Defossé; MM Davel; LO Bianchi \& TE Withington. 2013. Fire caused tree mortality in thinned Douglas-fir stands in Patagonia, Argentina. Int. J. Wildland Fire, 22:810-814.

Gonda, H. 2005. Efectos de la densidad sobre el crecimiento de un rodal mixto de pinos ponderosa y jeffrey en Neuquén. III Congreso Forestal Argentino-Latinoamericano. Corrientes, Argentina. Pp. 10.

Gonda, H \& J Lomagno. 1995. El raleo de plantaciones jóvenes de pino poderosa puede producir un importante volumen de materia prima comercial. IV Jornadas Forestales Patagónicas, vol. I. Asentamiento Universitario, San Martín de los Andes, Neuquén, Argentina. Pp. 299-305.

GonZÁlez, L. 2000. Consecuencias ambientales de la actividad ganadera: la desertificación. En: García, A y E Masón (comp.). El gran libro de la provincia de Santa Cruz. Milenio Ediciones / Alfa Centro Literario. España.

GonZÁLeZ, S. 2011. Estrategias de regeneración post-fuego en pastizales del Noroeste Patagónico: un enfoque experimental. Tesis doctoral. Universidad Nacional del Comahue. Bariloche, Argentina.

Greslebin, AG \& EM Hansen. 2010. Pathogenicity of Phytophthora austrocedrae on Austrocedrus chilensis and its relation with mal del ciprés in Patagonia. Plant Pathology, 59(4):604-612.

Gyenge, AG; ME Fernández; V Rusch; MM Sarasola \& T SCHLICHTER. 2010. Towards Sustainable Forestry Development in Patagonia: Truths and Myths of 
Environmental Impacts of Plantations with FastGrowing Conifers. Global Science Books. The Americas Journal of Plant Science and Biotechnology, 3:9-22.

Instituto Nacional de Estadísticas y Censos (INDEC). 2010. Censo Nacional de Población, Hogares y Viviendas 2001-2010. MECON, Buenos Aires. Argentina.

INFOR. 2014. Anuario Forestal 2014. Boletín estadístico Nro. 144. Instituto Forestal, Santiago, Chile. Pp. 166.

INTI. 1976. Congreso técnico latinoamericano sobre celulosa y papel. Trabajos. Biblioteca del Instituto Nacional de Tecnología Industrial (INTI), Buenos Aires. Argentina.

IRISARRI, JA \& JM MENDía. 1991. Reconocimiento de suelos y evaluación de la aptitud forestal de la región precordillerana de la Pcia. de Río Negro. CFI, Buenos Aires, Argentina. Pp. 61.

Irisarri, J; JM Mendia; C Roca; C Buduba; F Valenzuela; ET AL. 1995. Zonificación de las tierras para la forestación. Provincia del Chubut. Dirección General de Bosques y Parques de la Provincia del Chubut, Esquel, Chubut. Argentina.

JOVANOVSKI A; R MANFREDI \& P VILLENA. 2003. Evaluación de alternativas para aumentar la competitividad de la industria forestal de procesamiento primario en la provincia de Tierra del Fuego. Consejo Federal de Inversiones. Tierra del Fuego. Argentina.

Kimmins, JP. 2000. Residuals in forest ecosystems: sustainability and biodiversity issues. Pp: 29-38 en: Henry, CL; RB Harrison \& RK Bastian (eds.). The forest alternative: principles and practice of residuals use. College of Forest Resources, University of Washington, Seattle.

LANTSCHNER V \& V RUSCH. 2014. Efecto de las plantaciones forestales sobre la fauna en la Patagonia andina: ¿cómo compatibilizar la producción con la conservación? Producción Forestal, 8:12-14.

Licata, J; TG Pypker; M Weigandt; MH Unsworth; JE GYENGE; ET AL. 2011. Decreased rainfall interception balances increased transpiration in exotic ponderosa pine plantations compared with native cypress stands in Patagonia, Argentina. Ecohydrology, 4:83-93.

Loguercio, GA \& F Dececchis. 2006a. Forestaciones en la Patagonia Andina: potencial y desarrollo alcanzado. Parte 1. Patagonia Forestal, 1:1-4.

LOGUERCIO, GA \& F DECECCHIS. 2006b. Forestaciones en la Patagonia Andina: potencial y desarrollo alcanzado. Parte 2. Patagonia Forestal, 1:5-8.

Loguercio, GA; J Lencinas \& S Antequera. 2004. Bases Estratégicas para Proyectos de Forestación en la Patagonia como un Mecanismo para un Desarrollo Limpio. Estudio de Caso en la Provincia del Chubut. Proyecto Forestal de Desarrollo (SAGPyA) - CIEFAP, Esquel, Argentina.

Loguercio, GA; A Jovanovski; JC Molina \& P Pantaenius. 2008. Residuos de biomasa de forestaciones y aserraderos de la región Andina de las Provincias de Chubut y Neuquén. Evaluación preliminar de oferta. Publicación técnica. CIEFAP - No. 34. CIEFAP - JICA, Esquel, Argentina. Pp. 69.

Millenium Ecosystem Assessment (MEA). 2005. Ecosystems and Human Well-Being: Biodiversity Synthesis. World Resources Institute, Washington, DC.

Orellana, I. 2011. Efecto de borde de las plantaciones de Pseudotsuga menziesii sobre comunidades vegetales naturales del noroeste patagónico. Tesis Doctoral, Universidad del
Comahue. Bariloche, Argentina.

O'HARA, KL. 2001. The silviculture of transformation- a commentary. For. Ecol. Manage., 151:81-86.

Pauchard A; B Langdon \& E Peña. 2008. Potencial invasivo de Pseudotsuga menziesii (Mirb.) Franco en Bosques nativos del centro-sur de Chile: patrones y recomendaciones. Pp 89-114 en: Mujica, R; H Grosse \& B Muller-Using (eds.). Bosques seminaturales, una opción para la rehabilitación de bosques nativos degradados. Instituto Forestal, Valdivia. Chile.

Poder Ejecutivo Nacional (PEN). 1977. Ley 21695/77. Boletín Oficial Nro. 23802. Buenos Aires, Argentina.

PESCADOR, M\&S PERIs. 2014. Effects of Patagonian pine forestry on native breeding birds. For. Syst., 23(3):403-410.

Quinteros, CP. 2014. Grandes herbívoros en bosques de lenga (Nothofagus pumilio): uso espacio-temporal de los recursos y sus efectos sobre la regeneración y el sotobosque. Tesis Doctoral. Universidad Nacional del Comahue. Bariloche, Argentina

Rothkugel, M. 1916. Los Bosques Patagónicos. Oficina de Bosques y Yerbales. Talleres Gráficos del Ministerio de Agricultura de la Nación, Argentina.

SALVAdOR, G; MV FernándeZ \& G RoBLEs. 2006. Mercado de Madera para la Construcción proveniente de Plantaciones. Estudio Exploratorio en la Provincia del Chubut. Actas de I Reunión sobre Forestación en la Patagonia. Esquel, Chubut, Argentina. Del 25 al 27 abril de 2006.

Sarasola, M; VE Rusch; TM Schlichter \& CM GHERSA. 2006. Invasión de coníferas forestales en áreas de estepa y bosques de ciprés de la cordillera en la Región Andino Patagónica. Ecol. Austral, 16:143-156.

SAyDS. 2013. Comercio exterior de productos forestales 2011. Programa Nacional de Estadística Forestal. Dirección de Bosques, Secretaría de Ambiente y Desarrollo Sustentable. Buenos Aires, Argentina. Pp. 97.

Simberloff, D; MA Relva \& M NúÑEz. 2002. Gringos en el bosque: introduced tree invasion in a native Nothofagus/Austrocedrus forest. Biol. Invasions, 4: 35-53.

SORIANO, A. 1956. Aspectos ecológicos y pasturiles de la vegetación patagónica relacionados con su estado y capacidad de recuperación. Rev. Inv. Agr., 10:349-372.

SPF. 2014. Forestación en Uruguay. Productos forestales. Sociedad de Productores Forestales del Uruguay. www.spf.com.uy/forestacion-en-uruguay/productosforestales.

Subsecretaría de Bosques del Chubut (SSBCh). 2014. Estadísticas forestales. Esquel, Chubut. Argentina.

Urretavizcaya, MF; GE Defossé \& H Gonda. 2012. Effect of Sowing Season, Plant Cover, and Climatic Variability on Seedling Emergence and Survival in burned Austrocedurs chilensis forests. Restoration Ecol., 20(1):131-140.

URzúA, JD. 1991. Desarrollo de las plantaciones forestales del Neuquén y su aprovechamiento industrial. CIEFAP. Publicación Técnica 3, Esquel, Argentina.

Veblen, TT; T Kitzberger; E Raffaele; M Mermoz; ME GONZÁLEZ; ET AL. 2008. The historical range of variability of fires in the Andean-Patagonian Nothofagus forest region. Int. J. Wild. Fire, 17:724-741.

Wright, HA \& AW Bailey. 1982. Fire Ecology, United States and Southern Canada. Wiley, New York. 